\title{
EXACT PAIRS FOR THE IDEAL OF THE $K$-TRIVIAL SEQUENCES IN THE TURING DEGREES
}

\author{
GEORGE BARMPALIAS AND ROD G. DOWNEY
}

\begin{abstract}
The $K$-trivial sets form an ideal in the Turing degrees, which is generated by its computably enumerable (c.e.) members and has an exact pair below the degree of the halting problem. The question of whether it has an exact pair in the c.e. degrees was first raised in [MN06, Question 4.2] and later in [Nie09, Problem 5.5.8].

We give a negative answer to this question. In fact, we show the following stronger statement in the c.e. degrees. There exists a $K$-trivial degree $\mathbf{d}$ such that for all degrees $\mathbf{a}, \mathbf{b}$ which are not $K$-trivial and $\mathbf{a}>\mathbf{d}, \mathbf{b}>\mathbf{d}$ there exists a degree $\mathbf{v}$ which is not $K$-trivial and $\mathbf{a}>\mathbf{v}, \mathbf{b}>\mathbf{v}$. This work sheds light to the question of the definability of the $K$-trivial degrees in the c.e. degrees.
\end{abstract}

\section{INTRODUCTION}

The algebraic study of the Turing degrees has been a topic of considerable research in computability theory, ever since the establishment of degree theory as a research area in [KP54]. In this study, the ideals of this uppersemilattice are of particular interest. These are downward closed sets of degrees that also closed under the join operator. The recent study of algorithmic information theory by people in computability theory has brought forward a wealth of interactions between the two areas, including the discovery of a new ideal in the Turing degrees: the degrees of sequences with trivial initial segment complexity, the so-called $K$-trivial sequences. Since this discovery in [DHNS03, Nie05], the study of the $K$-trivial sequences and degrees has been established as a major area of research in the interface between computability theory and algorithmic information theory.

Issues of definability have been of special interest in the study of ideals in the Turing degrees. Such issues were already present in [KP54], where the notion of exact pairs of ideals was introduced. Two degrees $\mathbf{a}, \mathbf{b}$ form an exact pair of an ideal $\mathcal{C}$ in the Turing degrees if they are both upper bounds for the degrees in $\mathcal{C}$ and any degree below both $\mathbf{a}$ and $\mathbf{b}$ is in $\mathcal{C}$. By [KP54, Spe56] every ideal in the Turing degrees has an exact pair. By [Nie05] every $K$-trivial degree is bounded by

First version: May 18, 2012. This version: October 26, 2013.

2010 Mathematics Subject Classification. 03D25, 03D32, 68Q30.

Key words and phrases. Computably enumerable, Turing degrees, Kolmogorov complexity, $K$-trivial sets, exact pairs.

This research was partially done whilst the authors were visiting fellows at the Isaac Newton Institute for the Mathematical Sciences, Cambridge U.K., in the programme 'Semantics \& Syntax'. Barmpalias was supported by the Research fund for international young scientists number 613501-10236 from the National Natural Science Foundation of China, and an International Young Scientist Fellowship number 2010-Y2GB03 from the Chinese Academy of Sciences. Downey was supported by a Marsden grant of New Zealand. The authors wish to thank André Nies, Ted Slaman and Andy Lewis-Pye for helpful discussions. 
a computably enumerable (c.e. for short) $K$-trivial degree. Hence for the purpose of finding exact pairs for this ideal it suffices to consider its restriction to the c.e. degrees. This turns out to be a $\Sigma_{3}^{0}$ ideal, in the sense that the index set of its members is $\Sigma_{3}^{0}$. Moreover by [BN11] it has a c.e. upper bound that is strictly below the degree $\mathbf{0}^{\prime}$ of the halting problem (moreover, by [KS09] it has a low upper bound $\mathbf{b}$, which means that the halting problem relativized to $\mathbf{b}$ has degree $\mathbf{0}^{\prime}$ ). By [Sho81], such ideals have an exact pair strictly below $\mathbf{0}^{\prime}$. However it is well known that such an ideal may or may not have an exact pair in the c.e. degrees (this follows from the existence of branching and non-branching degrees that was established in [Lac66, Yat66]). Hence whether or not such an ideal has an exact pair in the c.e. degrees depends on the specific properties of it. The following question has come into focus.

Problem (Question 4.2 in [MN06] and Problem 5.5.8 in [Nie09]). Is there an exact pair for the ideal of the $K$-trivial sequences in the c.e. degrees?

The purpose of this paper is to give a negative answer to this question. In fact, our main result can be seen as a very strong negative answer to this question.

Theorem 1.1. There exists a $K$-trivial c.e. degree $\mathbf{d}$ with the following property. For each pair of c.e. degrees $\mathbf{a}, \mathbf{b}$ which are not $K$-trivial, there exists a c.e. degree $\mathbf{v}$ which is not $K$-trivial and $\mathbf{v}<\mathbf{a} \cup \mathbf{d}, \mathbf{v}<\mathbf{b} \cup \mathbf{d}$.

Here $\mathbf{a} \cup \mathbf{d}$ denotes the join (i.e. supremum) of the degrees $\mathbf{a}, \mathbf{d}$.

This theorem provides new and interesting information about the $K$-trivial sequences and their computational power. Moreover, as we elaborate in Section 2, it rests upon deeper information-theoretic properties that are specific to the $K$-trivial sequences, rather than some general property that this ideal happened to have. In contrast, the existence of a low bound of this ideal (another question from [MN06]) was obtained in [KS09] by observing that it satisfied a certain domination property, and proving that all ideals which share this property have a low bound.

We may obtain a negative answer to our problem by using some known properties of the $K$-trivial sequences.

Corollary 1.2. The ideal of the $K$-trivial sequences does not have an exact pair of c.e. degrees.

Proof. By [Nie02] there is no low c.e. upper bound for the $K$-trivial degrees. By [Nie05] every $K$-trivial degree is low. Therefore, if two c.e. degrees are an exact pair for the $K$-trivial degrees, then both of them are not $K$-trivial. The corollary now follows directly from Theorem 1.1.

Note that the proof of Corollary 1.2 rests on the following weak (and nonuniform) version of Theorem 1.1: 'given a pair $\mathbf{a}, \mathbf{b}$ of c.e. degrees which are not $K$-trivial, there exists a $K$-trivial c.e. degree $\mathbf{d}$ and c.e. degree $\mathbf{v}$ which is not $K$-trivial such that $(\mathbf{d} \leq \mathbf{a} \wedge \mathbf{d} \leq \mathbf{b}) \rightarrow(\mathbf{v} \leq \mathbf{a} \wedge \mathbf{v} \leq \mathbf{b})$.

The following fact is a direct consequence of the splitting theorem from [Bar11, Section 5] and [Ste11, Chapter 2]. It shows that by replacing $\mathbf{v}<\mathbf{a} \cup \mathbf{d}, \mathbf{v}<\mathbf{b} \cup \mathbf{d}$ with $\mathbf{v} \leq \mathbf{a} \cup \mathbf{d}, \mathbf{v} \leq \mathbf{b} \cup \mathbf{d}$ in Theorem 1.1 we obtain an equivalent statement.

Proposition 1.3. If $\mathbf{c}$ is a c.e. degree which is not $K$-trivial then there exist c.e. degrees $\mathbf{a}<\mathbf{c}$ and $\mathbf{b}<\mathbf{c}$ which are not $K$-trivial and $\mathbf{c}=\mathbf{a} \cup \mathbf{b}$. 
Since there exists a $\Delta_{2}^{0}$ exact pair for the $K$-trivial degrees, the phenomenon described in Theorem 1.1 is specific to c.e. sets. The following observation contrasts Proposition 1.3 and confirms this intuition from a different angle.

Proposition 1.4. There exists a degree $\mathbf{x}<\mathbf{0}^{\prime}$ which is not $K$-trivial and for every $K$-trivial degree $\mathbf{d}$, the only c.e. degrees that are computable from $\mathbf{x} \cup \mathbf{d}$ are also computable from $\mathbf{d}$.

Proof. A degree that is 1-generic relative to every $K$-trivial degree has the desired properties, but is not necessarily below $\mathbf{0}^{\prime}$. Moreover no 1 -generic set has degree $\mathbf{0}^{\prime}$. Hence it suffices to show that there exists a degree that is 1-generic relative to every $K$-trivial degree and computable from the halting problem. This follows from the fact (see [KS09]) that there exists a function that is computable from the halting problem and dominates all partial computable functions relative to any $K$-trivial set.

The proof of Theorem 1.1 rests on a few facts about $K$-trivial sequences and initial segment Kolmogorov complexity. We present these, along with their use in the proof, in Section 2. Some background on Kolmogorov complexity and $K$-trivial sequences that is directly relevant to our result is given in Section 2.1. For background material on computability theory we refer to [Odi89]. The main property of Kolmogorov complexity that is used in the proof of Theorem 1.1 is discussed in Section 2.3. It is a result from [Bar13] which roughly says that any two c.e. sets of nontrivial initial segment complexity must have common lengths in their characteristic sequences where their complexity rises simultaneously. Our proof is essentially a derivation of Theorem 1.1 from this result. This route reduces the complexity of the main construction and results in a transparent presentation.

Two more tools from Kolmogorov complexity are used in order to reduce the calculations further and avoid the dynamic construction of machines in the main construction. The first is the use of Solovay functions to express $K$-triviality, which is based on [BD09, BMN11]. The second one is the standard computable invariance property that is intrinsic to most notions in Kolmogorov complexity. Both of these tools are discussed in Section 2.2. Section 2.5 provides the exact form of the result from [Bar13] that will be used in the main argument, which is given in Section 3. These few preparatory steps (including the formulation of a sufficient set of requirements in Section 3.1) reduce the main argument to the simple construction and verification of Sections 3.3 and 3.4.

\section{Preliminary facts}

In this section we provide a number of notions and results that are needed for the proof of Theorem 1.1. Some of these facts are known, while others are original.

2.1. Background on Kolmogorov complexity and $K$-trivial sequences. A standard measure of the complexity of a finite string was introduced by Kolmogorov in [Kol65] (an equivalent approach was due to Solomonoff [Sol64]). The basic idea behind this approach is that simple strings have short descriptions relative to their length while complex or random strings are hard to describe concisely. Kolmogorov (and Solomonoff) formalized this idea using the theory of computation. In this context, Turing machines play the role of our idealized computing devices, and we assume that there are Turing machines capable of simulating any mechanical 
process which proceeds in a precisely defined and algorithmic manner. Programs can be identified with binary strings.

A string $\tau$ is said to be a description of a string $\sigma$ with respect to a Turing machine $M$ if this machine halts when given program $\tau$ and outputs $\sigma$. Then the Kolmogorov complexity of $\sigma$ with respect to $M$ (denoted by $K_{M}(\sigma)$ ) is the length of its shortest description with respect to $M$. It can be shown that there exists an optimal machine $V$, i.e. a machine which gives optimal complexity for all strings, up to a certain constant number of bits. This means that for each Turing machine $M$ there exists a constant $c$ such that $K_{V}(\sigma)<K_{M}(\sigma)+c$ for all finite strings $\sigma$. Hence the choice of the underlying optimal machine does not change the complexity distribution significantly and the theory of Kolmogorov complexity can be developed without loss of generality, based on a fixed underlying optimal machine $U$.

When we come to consider the initial segment complexity of infinite strings, it becomes important to consider machines whose domain satisfies a certain condition; the machine $M$ is called prefix-free if it has prefix-free domain (which means that no program for which the machine halts and gives output is an initial segment of another). Prefix-free complexity was introduced by Levin [Lev73] and Chaitin [Cha75]. Similarly to the case of ordinary Turing machines, there exists an optimal prefix-free machine $U$ so that for each prefix-free machine $M$ the complexity of any string with respect to $U$ is up to a constant number of bits larger than the complexity of it with respect to $M$. We let $K$ denote the prefix-free complexity with respect to a fixed optimal prefix-free machine. Order the binary strings first by length and then lexicographically. This standard ordering of the strings induces a computable bijections between $\mathbb{N}$ and the binary strings. Under this bijection we may identify numbers and strings. In this sense we may talk about the complexity $K(n)$ of a number $n$ as being the complexity of the string that is represented by $n$.

The original motivation behind Kolmogorov complexity was a mathematical definition of random infinite sequences. Kolmogorov's idea was that these should be infinite sequences with very complex initial segments. Based on this intuition, Levin [Lev73] and Chaitin [Cha75] gave a robust definition of randomness for infinite binary sequences, which coincided with Martin-Löf randomness (already defined in [ML66]). They called $X$ random if $\exists c \forall n, K\left(X \uparrow_{n}\right) \geq n-c$. In other words, $X$ is random if its initial segments cannot be 'compressed' (i.e. be described more concisely) by more than a constant number of bits.

In this paper we are concerned with the other end of the spectrum: sequences with trivial initial segment complexity. These are sequences whose initial segments are very highly compressible, in the sense that they have very short descriptions.

Definition 2.1 ( $K$-trivial sequences). An infinite binary sequence $X$ is called $K$ trivial if $\exists c \forall n, K\left(X \uparrow_{n}\right) \leq K(n)+c$.

Here $K(n)$ denotes the complexity of the number $n$. It follows from the basic properties of Kolmogorov complexity that $K(n)$ and $K\left(0^{n}\right)$ are equal up to an additive constant. Hence the first $n$ bits of a $K$-trivial sequence have the same complexity as the sequence $0^{n}$. By identifying subsets of $\mathbb{N}$ with their characteristic sequence we can also talk about $K$-trivial sets of numbers. Chaitin drew some attention to $K$ trivial sets by noticing that they are computable from the halting problem and by asking whether they are all computable. Solovay [Sol75] produced the first example of a noncomputable K-trivial set. The work in [DHNS03] signaled a renewed interest 
on this notion and initiated a deeper study of $K$-triviality which revealed surprising connections between initial segment complexity and classical computability. For example, Hirschfeldt and Nies showed in [Nie05] that $K$-triviality is downward closed under Turing computation. Moreover the K-trivial sets form an ideal in the Turing degrees, which is generated by its c.e. members (in the sense that every $K$-trivial set is computable by a c.e. $K$-trivial set).

2.2. Solovay functions and computable invariance. Building on work from [Sol75], the following characterisation of $K$-trivial sets was given in [BD09].

There exists a computable function $g: \mathbb{N} \rightarrow \mathbb{N}$ such that

$$
\begin{aligned}
& \text { (*) } \quad X \text { is } K \text {-trivial } \Longleftrightarrow \exists c \forall n\left(K\left(X \uparrow_{n}\right) \leq g(n)+c\right) \\
& \text { for all sets } X \text { and also } \sum_{n} 2^{-g(n)} \text { is a random real. }
\end{aligned}
$$

Here by a random real we mean a real number in $(0,1)$ whose binary expansion is a random sequence. Later it was demonstrated in [BMN11] that the functions $g$ of (2.1) are exactly the computable tight upper bounds of the Kolmogorov function $K(n)$, in the sense for some constant $c$ we have $K(n) \leq g(n)+c$ for all $n$ and $g(t) \leq K(t)+c$ for infinitely many $t$. These functions were called Solovay functions. By [BD09, HKM09] a computable function $g$ is a Solovay function if and only if $\sum_{n} 2^{-g(n)}$ is a random real.

Note that (2.1) replaces a non-computable component in the definition of $K$ triviality (namely $K(n)$ ) with a computable function. In certain situations this allows for a simplification of the calculations involved in arguments about the $K$ trivial sets. This is the case with the proof of Theorem 1.1.

Before we fix a Solovay function for use in the proof of Theorem 1.1, let us discuss a few basic facts about Solovay functions. We start with a certain computable invariance that is common in many notions related to Kolmogorov complexity.

Proposition 2.2 (Computable invariance of Solovay functions). Let $f$ be a Solovay function and let $m_{i}: \mathbb{N} \rightarrow \mathbb{N}$ be a computable increasing sequence. Then $i \mapsto f\left(m_{i}\right)$ is a Solovay function.

Proof. Since $\left(m_{i}\right)$ is increasing and computable, $\exists c \forall i, K(i) \leq K\left(m_{i}\right)+c$. Hence $i \mapsto f\left(m_{i}\right)$ is a computable upper bound of $K(i)$. Also $\exists b \forall i, K\left(m_{i}\right) \leq K(i)+b$. So $i \mapsto f\left(m_{i}\right)$ is a computable tight upper bound of $K(i)$ and the proposition follows from the characterization of Solovay functions from [BMN11].

It is well known (e.g. see [Nie09, Exercise 5.2.9]) that if $\left(m_{i}\right)$ is a computable increasing sequence then $\exists b \forall i, K\left(X \uparrow_{i}\right) \leq K\left(X \uparrow_{m_{i}}\right)+b$ The following fact is a direct consequence of the above observation and Proposition 2.2.

Proposition 2.3 ( $K$-triviality in terms of Solovay functions). Let $f$ be a Solovay function and let $m_{i}: \mathbb{N} \rightarrow \mathbb{N}$ be a computable increasing sequence. A set $X$ is $K$-trivial if and only if $\exists c \forall i, K\left(X \uparrow m_{i}\right) \leq f\left(m_{i}\right)+c$.

The following observation is a direct consequence of the fact that $\sum_{t} 2^{-f(t)}$ is noncomputable when $f$ is a Solovay function.

Proposition 2.4 (Accumulation of weight in Solovay functions). If $\left(m_{i}\right)$ is a computable increasing sequence and $f$ is a Solovay function then for every $k$ there exist infinitely many $n$ such that $\sum_{t>m_{n}} 2^{-f(t)}>\frac{1}{n-k-1}$. 
In the following sections, we fix a computable function $g$ as in (2.1) and use $(\star)$ as a characterisation of $K$-triviality. A c.e. real is a real that is the limit of a computable non-decreasing sequence of rationals. Define

$$
\Omega=\sum_{i} 2^{-g(i)} \text { and } \quad \Omega_{n}=\sum_{i<n} 2^{-g(i)} .
$$

The letter $\Omega$ is often used to denote the halting probability of a universal prefix-free machine. Since these numbers coincide with the random c.e. reals (e.g. see [DH10, Section 9.2] or the original references [KS01, CHKW01]) we may use it in order to denote $\sum_{n} 2^{-g(n)}$. Without loss of generality we may assume that $\Omega<1 / 4$. A set $X$ is called low for $\Omega$ if $\Omega$ is random relative to $X$. Here are some facts about this class of sets that we are going to use in this article (for more information on this topic we refer to [Nie09, Section 8.1]). The low for $\Omega$ sets form a proper superclass of the $K$-trivial sets. Inside the $\Delta_{2}^{0}$ sets, the low for $\Omega$ sets coincide with the $K$ trivial sets. Let us fix the computable bijection $(m, n) \mapsto\langle m, n\rangle$ that we used, since we will need to refer to it in later section

In this and the previous background section we focused on aspects of $K$-triviality that are directly relevant to the proof of Theorem 1.1. For a more thorough presentation of the research area algorithmic randomness and complexity-theoretic weakness we refer to the monographs [Nie09, DH10], while [LV97] is a standard reference for the more general theory of Kolmogorov complexity.

2.3. Common complexity in pairs of c.e. sets of nontrivial complexity. Much of the excitement about the $K$-trivial sequences comes from the fact that they provide an ideal platform for the study of the interaction between the information that can be coded into an infinite binary sequence and the complexity of its initial segments. The latter has been a primary focus of research in the interface between computability theory and Kolmogorov complexity. The fact that there are noncomputable $K$-trivial sequences showed that one can code nontrivial information into a sequence without increasing the complexity of its initial segments. A limitation to this phenomenon was revealed in [DHNS03] where it was shown that $K$-trivial sequences cannot compute the halting problem (in other words, they are not Turing complete). In contrast, there are Turing complete sequences of arbitrarily low nontrivial prefix-free initial segment complexity. More precisely, in [Bar13] it was shown that for every c.e. set $A$ which is not $K$-trivial, there exists a Turing complete c.e. set $V$ of lower complexity, i.e. such that $\exists c \forall n, K\left(V \uparrow_{n}\right) \leq K\left(A \uparrow_{n}\right)+c$. This was also generalized for the case of any finite collection $A_{i}, i<k$ of c.e. sets which are not $K$-trivial, producing a Turing complete c.e. set $V$ such that $\exists c \forall n \forall i<k, K\left(V \uparrow_{n}\right) \leq K\left(A_{i} \uparrow_{n}\right)+c$. A consequence this fact is (see [Bar13, Corollary 1.7]) is that

if $A, B$ are c.e. sets which are not $K$-trivial, then for each $c$ there exists $n$ such that $\min \left\{K\left(A \uparrow_{n}\right), K\left(B \uparrow_{n}\right)\right\}>K(n)+c$.

This fact is the crux of the proof of Theorem 1.1. It says that any pair of c.e. sets of nontrivial initial segment prefix-free complexity exhibit common lengths of nontrivial prefix-free complexity. It is just one of a series of results which indicate that any two c.e. sets of non-trivial initial segment complexity have some kind of common complexity, or even information. In view of the existence of minimal pairs in the c.e. Turing degrees (a classic result from [Lac66]), such information is not 
common in the terms of the Turing reducibility but in terms of weaker measures of relative complexity. See [Bar13, Theorem 1.2] and [Bar10, Theorem 1.3].

Instead of a direct proof, we have chosen to derive Theorem 1.1 as a consequence of (2.3). This route reduces the bulk of the proof to the rather simple construction and verification of Section 3. We note that even the proof of (2.3) from [Bar13] is not direct (strictly speaking) in the sense that it rests on the nontrivial result from [DHNS03] that Turing complete sets are not $K$-trivial.

2.4. Construction of prefix-free machines. A (rather simple) direct construction of a prefix-free machine will be used in Section 2.5. There are certain notions and tools associated with such constructions, which are standard in the arguments employed in algorithmic randomness and also relate to the main argument of Section 3. We briefly discuss them. The weight of a prefix-free set $S$ of strings, denoted $\operatorname{wgt}(S)$, is defined to be the sum $\sum_{\sigma \in S} 2^{-|\sigma|}$. The weight of a prefix-free machine $M$ is defined to be the weight of its domain and is denoted $\operatorname{wgt}(M)$. Prefix-free machines are most often built in terms of request sets. A request set $L$ is a set of pairs $\langle\rho, \ell\rangle$ where $\rho$ is a string and $\ell$ is a positive integer. A 'request' $\langle\rho, \ell\rangle$ represents the intention of describing $\rho$ with a string of length $\ell$. We define the weight of the request $\langle\rho, \ell\rangle$ to be $2^{-\ell}$. We say that $L$ is a bounded request set if the sum of the weights of the requests in $L$ is less than 1 . This sum is the weight of the request set $L$ and is denoted by wgt $(L)$.

The Kraft-Chaitin theorem (see e.g. [DH10, Section 2.6]) says that for every bounded request set $L$ which is c.e., there exists a prefix-free machine $M$ with the property that for each $\langle\rho, \ell\rangle \in L$ there exists a string $\tau$ of length $\ell$ such that $M(\tau)=\rho$. Hence the dynamic construction of a prefix-free machine can be reduced to a mere description of a corresponding c.e. bounded request set.

A function is called right-c.e. if it has a computable non-increasing approximation. Recall that a c.e. real is a real that is the limit of a computable non-decreasing sequence of rationals. Note that c.e. sets are c.e. reals but the converse does not hold. The Kraft-Chaitin theorem also implies that the definition of a prefix-free machine $N$ may be reduced (as far as the function $n \mapsto K_{N}(n)$ is concerned) to a definition of a right-c.e. function $h$ such that $\sum_{n} 2^{-h(n)}<1$. Indeed, given such a function $h$ we may define $K_{N}=h$. Then the Kraft-Chaitin theorem guarantees that such a machine $N$ exists. This useful method of defining prefix-free machines (when we are only concerned in the corresponding complexity function) will be used in several proofs in this paper, starting with a proof in the following section. The prefix relation amongst finite or infinite strings is denoted by $\prec$.

2.5. Modulus functions of c.e. sets and $K$-triviality. We use the following notion of 'modulus of convergence' which is associated with the enumeration of a set or the monotone approximation to a real.

Definition 2.5 (Modulus functions of c.e. sets). Let $A$ be a c.e. set (or real) with a computable enumeration $(A[s])$. The modulus function $n \mapsto a(n)$ of $A$ maps each $n$ to $\langle n, s\rangle$ where $s$ is the least stage such that $A[s] \Gamma_{n} \prec A$ and $s>n$.

Note that the modulus function of a c.e. set $A$ always refers to a particular computable enumeration $(A[s])$ of it. In this paper all c.e. sets will be given via a certain computable enumeration of them. Hence we may talk about the modulus function of a given c.e. set (suppressing the corresponding computable enumeration) without 
causing confusion. This also means that the modulus function of a c.e. sets comes automatically with a computable monotone approximation. Moreover according to Definition 2.5, the image $a(n)$ of the modulus function of a c.e. set encodes $n$. This property will be used in the proof of Lemma 2.10.

Modulus functions and $K$-triviality are related, as we show in this section. We start with a result which says that functions that are computed from $K$-trivial sets do not speed up the canonical computable approximation to $\Omega$. Without extra effort, we prove this for the larger class of low for $\Omega$ sets. This result is not needed for the proof of Theorem 3 but it gives a pleasing characterization of the c.e. $K$ trivial sets which we present in Corollary 2.9.

Lemma 2.6 (Low for $\Omega$ functions are slow growing). If $A$ is low for $\Omega$ and $f \leq_{T}$ then there exists a constant $c$ such that $\Omega-\Omega_{n}<2^{c} \cdot\left(\Omega-\Omega_{f(n)}\right)$ for all $n$.

Proof. Without loss of generality we may assume that $f$ is increasing. Indeed, otherwise we may consider $f^{\prime}(n)=\max _{i \leq n} f(n)+1$ and since $\Omega-\Omega_{f(n)}>\Omega-\Omega_{f^{\prime}(n)}$ the lemma about $f^{\prime}$ implies the lemma about $f$.

Define a Martin-Löf test $\left(V_{n}\right)$ relative to $A$ as follows. At stage $s+1$, do the following for each $n<s$. If $\Omega_{f(s)} \in V_{n}[s]$ do nothing. Otherwise let $t_{n}[s]$ be the last stage since we put something into $V_{n}$ (and $t_{n}[s]=0$ if such a stage does not exist) and put the interval $\left(\Omega_{f(s)}, \Omega_{f(s)}+2^{-n} \cdot\left(\Omega_{s}-\Omega_{t_{n}[s]}\right)\right)$ into $V_{n}$.

Clearly $\mu\left(V_{n}\right) \leq 2^{-n} \cdot \Omega$. Moreover $\left(V_{n}\right)$ is uniformly c.e. in $A$. Hence $\left(V_{n}\right)$ is a Martin-Löf test relative to $A$. Since $A$ is low for $\Omega$, there exists $n$ such that $\Omega \notin V_{n}$. Let $\left(s_{i}\right)$ be an increasing enumeration of the stages where an enumeration occurred in $V_{n}$ and note that there are infinitely many such stages. Then $\Omega_{f\left(s_{i+1}\right)}-\Omega_{f\left(s_{i}\right)}>$ $2^{-n} \cdot\left(\Omega_{s_{i}}-\Omega_{s_{i-1}}\right)$ for all $i>0$. Hence for each $i>0$ we have $\Omega-\Omega_{s_{i-1}}<$ $2^{n} \cdot\left(\Omega-\Omega_{f\left(s_{i}\right)}\right)$. Hence for each $i>0$ and each $t \in\left[s_{i-1}, s_{i}\right)$ we have

$$
\Omega-\Omega_{t} \leq \Omega-\Omega_{s_{i-1}}<2^{n} \cdot\left(\Omega-\Omega_{f\left(s_{i}\right)}\right) \leq 2^{n} \cdot\left(\Omega-\Omega_{f(t)}\right) .
$$

Hence for each $t$ we have $\Omega-\Omega_{t} \leq 2^{n} \cdot\left(\Omega-\Omega_{f(t)}\right)$ as required.

We do not know if the converse of Lemma 2.6 holds, thereby giving a characterization of the low for $\Omega$ sets. The interested reader may consult two other characterizations of this class that were obtained in [Mil10] and [BL11] respectively. We are able to prove that this equivalence holds inside the class of $\Delta_{2}^{0}$ sets. We present this result later in this section, in the form of Corollary 2.9.

The following notion of movable markers is implicit in many recursion-theoretic constructions. We isolate it since it can be used in order to elegantly describe the enumeration of a c.e. sets and prove Proposition 2.11, which is the ultimate goal of this section.

Definition 2.7 (Movable markers). A function $(n, s) \mapsto m_{n}[s]$ is a system of movable markers if it is non-decreasing in $n, s$ and if $m_{n}[s] \neq m_{n}[s+1]$ then $m_{n}[s+1]>s$.

According to the above definition, a movable marker need not be convergent.

Lemma 2.8 (Complexity of movable markers). Let $m_{n}[s]$ be a computable system of movable markers such that $\Omega-\Omega_{n}<2^{c} \cdot\left(\Omega-\Omega_{m_{n}}\right)$ for some constant $c$ and all $n$, where $m_{n}=\lim _{s} m_{n}[s]$. Then there exists $d$ such that $K\left(m_{n}\right) \leq K(n)+d$ for all $n$. 
Proof. Assume that $\Omega-\Omega_{n}<2^{c} \cdot\left(\Omega-\Omega_{m_{n}}\right)$ for some constant $c$ and all $n$. Then for all $n$ the limit $m_{n}$ is finite. Let $g$ be the Solovay function from (2.2). It suffices to define a prefix-free machine $N$ such that $K_{N}\left(m_{n}\right) \leq g(n)+c$ for all $n$. Let $k_{s}$ be the largest number such that $K_{N}\left(m_{n}\right)[s] \leq g(n)+c$ for all $n<k_{s}$. Then $k_{s}$ is defined for all $s$ and by the properties of the following construction, $k_{s} \leq s$ for all $s$. The following construction defines $N$, along with a partition $\left(I_{s}\right)$ of the set of stages which will be used in order to count the weight of $N$ in the verification. Let $I_{0}=\emptyset$.

At each stage $s+1$ we first check if $m_{i}[s] \neq m_{i}[s+1]$ for some $i<k_{s}$. If not, then we enumerate an $N$-description of $m_{k_{s}}[s]$ of length $g\left(m_{k_{s}}[s]\right)+c$ and say that this enumeration is primary. In this case we also define $I_{s+1}=\emptyset$. Otherwise we search for a stage $p>s+1$ such that

$$
\Omega_{m_{r}[p]}-\Omega_{r}<2^{c} \cdot\left(\Omega_{p}-\Omega_{m_{r}[p]}\right)
$$

where $r$ is the least such that $m_{r}$ moved during the stages in $(s, p]$ (note that $\left.r<k_{s}\right)$. By the hypothesis, such a stage $p$ exists. For each $i \in(s, p)$ let $I_{i}=\emptyset$. Define $I_{p}=\left[m_{r}[p], p\right]$. Note that by Definition 2.7 we have $m_{r}[p] \geq s+1$, so $m_{r}[p]$ is greater than all numbers in the intervals $I_{i}$ for $i<p$. By (2.4) we have

$$
\sum_{r \leq i<k_{s}} 2^{-g(i)}<2^{c} \cdot \sum_{i \in I_{p}} 2^{-g(i)}
$$

For each $i \in\left[r, k_{s}\right)$ enumerate an $N$-description of $m_{i}$ of length $g(i)+c$. We say that this enumeration of $N$-descriptions is secondary. Say that the stages in $(s, p]$ are dormant and go to stage $p+1$.

By the construction, the intervals $I_{i}$ are pairwise disjoint. Moreover the weight of the $N$-descriptions that correspond to primary enumerations amount to weight at most $\sum_{i} 2^{-g(i)}<\Omega$ because $\left(k_{s}\right)$ is non-decreasing on the stages where primary enumerations occur. At each stage $p$ where a secondary enumeration of $N$-descriptions takes place, by (2.5) the weight of these descriptions is bounded by $\sum_{i \in I_{p}} 2^{-g(i)}$. Hence the total weight of the $N$-descriptions that correspond to secondary enumerations is bounded by

$$
\sum_{p} \sum_{i \in I_{p}} 2^{-g(i)}=\Omega
$$

Hence the total weight of $N$ is bounded by $2 \cdot \Omega<1$. So $N$ is a prefix-free machine. By the construction, $k_{s} \rightarrow \infty$ as $s \rightarrow \infty$ and (through the secondary enumerations) we also have $K_{N}\left(m_{n}\right) \leq g(n)+c$.

We note that by the same argument as in the proof of Lemma 2.6, the converse of Lemma 2.8 is true. The following characterization is not needed for the proof of Theorem 1.1 but it is worth mentioning. It says that a c.e. set (or real) is $K$-trivial if and only if the monotone approximations to $\Omega$ that it can provide are no better than those that can be provided by a computable function.

Corollary 2.9 (Characterisation of $K$-trivial c.e. reals). Given a c.e. set (or real) $A$ the following are equivalent:

(a) $A$ is $K$-trivial;

(b) for all functions $f \leq_{T} A$ we have $\exists c \forall n \Omega-\Omega_{n}<2^{c} \cdot\left(\Omega-\Omega_{f(n)}\right)$;

(c) $\exists c \forall n \Omega-\Omega_{n}<2^{c} \cdot\left(\Omega-\Omega_{a(n)}\right)$. 
where $(a(n))$ is the modulus of its computable enumeration (or monotone approximation, in the case of c.e. reals).

Proof. The implication $(\mathrm{a}) \Rightarrow(\mathrm{b})$ follows from Lemma 2.6 and the fact that $K$ trivial sets are low for $\Omega$. The implication $(\mathrm{b}) \Rightarrow(\mathrm{c})$ is trivial. For the remaining implication $(\mathrm{c}) \Rightarrow(\mathrm{a})$, assume that there exists a constant $c$ such that $\Omega-\Omega_{n}<$ $2^{c} \cdot\left(\Omega-\Omega_{a(n)}\right)$ for all $n$. Note that $(a(n)[s])$ is a computable system of movable markers according to Definition 2.7. Hence by Lemma 2.8 there exists a constant $c_{0}$ such that $K(a(n)) \leq K(n)+c_{0}$. On the other hand there exists a constant $c_{1}$ such that $K\left(A \uparrow_{n}\right) \leq K(a(n))+c_{1}$ for each $n$. Hence there exists a constant $p$ such that $K\left(A \uparrow_{n}\right) \leq K(n)+p$ for all $n$, which means that $A$ is $K$-trivial.

The following observation is the last step that we need in order to derive the main result of this section which is Proposition 2.11; it connects the complexity of certain system of movable markers with the initial segment complexities of a pair of c.e. sets.

Lemma 2.10. Let $A, B$ be c.e. sets (or reals), let $(a(n)),(b(n))$ be their modulus functions and let $d(n)=\min \{a(n), b(n)\}$. If there exists $p$ such that $K(d(n)) \leq$ $K(n)+p$ for all $n$ then there exists $c$ such that $\min \left\{K\left(A \uparrow_{n}\right), K\left(B \uparrow_{n}\right)\right\}<K(n)+c$ for all $n$.

Proof. It suffices to show that there exists a prefix-free machines $M_{a}, M_{b}$ such that $\min \left\{K_{M_{a}}\left(A \uparrow_{n}\right), K_{M_{b}}\left(B \uparrow_{n}\right)\right\} \leq K\left(d_{n}\right)$ for all $n$. Consider $M_{a}$ which, on input $\sigma$ operates as follows. First it waits until $U(\sigma) \downarrow=\langle n, t\rangle$ for some $t, n$ (where $U$ is the optimal machine). Then it defines $M_{a}(\sigma)=A_{t} \Gamma_{n}$. Similarly, on input $\sigma$ machine $M_{b}$ waits until $U(\sigma) \downarrow=\langle n, t\rangle$ for some $t$, nand then defines $M_{b}(\sigma)=B_{t} \uparrow_{n}$. Clearly $M_{a}, M_{b}$ are prefix free (as they have the same domain as $U$ ). Moreover, for each $n$ let $t_{n}$ be such that $d_{n}=\langle n, t\rangle$. Since $d(n)=a(n)$ or $d(n)=b(n)$ for each $n$, we have $A_{t_{n}} \uparrow_{n}=A \uparrow_{n}$ or $B_{t_{n}} \uparrow_{n}=B \uparrow_{n}$ for each $n$. Hence for each $n$ we have $K_{M_{a}}\left(A \uparrow_{n}\right) \leq K(d(n))$ or $K_{M_{b}}\left(B \uparrow_{n}\right) \leq K(d(n))$, which completes the proof.

Proposition 2.11 (Tool for the main construction). Let $A, B$ be c.e. sets (or reals), let $(a(n)),(b(n))$ be their modulus functions and let $d(n)=\min \{a(n), b(n)\}$. If $A, B$ are not $K$-trivial then for each $c$ there exists $n$ such that $\Omega-\Omega_{d(n)}<2^{-c} \cdot\left(\Omega-\Omega_{n}\right)$.

Proof. This is a direct consequence of Lemma 2.8, Lemma 2.10 and (2.3).

Note that the conclusion $\Omega-\Omega_{d(n)}<2^{-c} \cdot\left(\Omega-\Omega_{n}\right)$ can be written as

$$
\sum_{i>d(n)} 2^{-g(i)}<2^{-c} \cdot \sum_{i>n} 2^{-g(i)}
$$

by (2.2). This is the form that we are going to use when we formulate the requirements for the proof of Theorem 1.1 in Section 3.1.

\section{Proof of Theorem 1.1}

We wish to construct a c.e. set $D$, whose Turing degree $\mathbf{d}$ meets the conditions of Theorem 1.1. We formulate a sufficient set of requirements for $D$ in Section 3.1 and give the specifics of the construction in Section 3.2. We conclude with the formal construction in Section 3.3 and the verification of the requirements in Section 3.4. 
3.1. Requirements for the construction of $D$. Let $U$ be the universal prefixfree machine which underlies the prefix-free Kolmogorov complexity function, i.e. such that $K=K_{U}$. We may assume that $\operatorname{wgt}(U)<2^{-4}$. Also let $\left(A_{e}, B_{e}\right)$ be an effective list of all pairs of c.e. sets. Note that the sets $A_{e}, B_{e}$ are given via specific computable enumerations that are provided by a fixed universal Turing machine. The sets $A_{e}, B_{e}$ correspond to guesses about representatives of the degrees $\mathbf{a}, \mathbf{b}$ of Theorem 1.1. For each pair $\left(A_{e}, B_{e}\right)$ let $a_{e}, b_{e}$ denote the corresponding modulus functions. Moreover let $a_{e}[s], b_{e}[s]$ denote their approximations at stage $s$. In particular, $a_{e}(n)[s]$ is $n$ if $s \leq n$ and the least stage $t>n$ with $t \leq s$ such that $\left.A[t]\right|_{n} \prec A[s]$ otherwise; similarly for $b_{e}(n)[s]$. Let $(i, j) \mapsto\langle i, j\rangle$ be a standard computable increasing (in both arguments) pairing function and define $\mathbb{N}^{[k]}=\{\langle k, n\rangle \mid n \in \mathbb{N}\}$.

We define a version of the parameter $\min \left\{a_{e}(n), b_{e}(n)\right\}$ which can be treated dynamically (at any stage of the construction) as a number that is eligible for enumeration into the set $D$ that will be constructed. Define $d_{e}(n)[s]$ to be the least number in $\mathbb{N}^{[\langle e, n\rangle]}-D[s]$ which is larger than $\min \left\{a_{e}(n)[s], b_{e}(n)[s]\right\}$. Moreover let $d_{e}(n)=\lim _{s} d_{e}(n)[s]$. The parameters $a_{e}(n)[s], b_{e}(n)[s], d_{e}(n)[s]$ can be seen as movable markers on $\mathbb{N}$. Moreover a direct consequence of their definition is that they always move monotonically, i.e. $a_{e}(n)[s] \leq a_{e}(n)[s+1]$ and similarly for $b_{e}(n)[s], d_{e}(n)[s]$.

We will define a $K$-trivial c.e. set $D$ and a sequence of c.e. sets $\left(V_{e}\right)$ such that the following conditions are met.

$$
R_{e}: V_{e} \leq_{T} A_{e} \oplus D \wedge V_{e} \leq_{T} B_{e} \oplus D .
$$

We will also ensure the following condition on $V_{e}$.

$$
P_{e} \text { : If } A_{e}, B_{e} \text { are not } K \text {-trivial then } V_{e} \text { is not } K \text {-trivial. }
$$

These conditions on $D,\left(V_{e}\right)$ are sufficient for the proof of Theorem 1.1. Let $g$ be a fixed Solovay function, i.e. a function satisfying (2.1), for the duration of this proof. Without loss of generality we may assume that $\sum_{i} 2^{-g(i)}<2^{-4}$. We may split each condition $P_{e}$ into more elementary conditions $P_{e k t}^{*}$. Let $(k, i) \mapsto n_{k}(i)$ be a computable function such that $n_{k}(i)<n_{k}(i+1)$ and $n_{k}(i) \in \mathbb{N}^{[k]}$. In Section 3.2 we will define a specific such function, but at this point we may express $P_{\text {ekt }}^{*}$ in terms of any fixed such choice. We may write $n_{k t}$ to denote $n_{k}(t)$ in the interest of space.

$$
P_{e k t}^{*}:\left(\sum_{i>d_{e}\left(n_{k t}\right)} 2^{-g(i)}<2^{-e-k} \cdot \sum_{i>n_{k t}} 2^{-g(i)}\right) \Rightarrow K\left(V_{e}\left\lceil n_{k t}\right)>g\left(n_{k t}\right)+k .\right.
$$

We let $P_{e k}^{*}$ denote the conjunction of all $P_{e k t}^{*}, t \in \mathbb{N}$. We verify that the satisfaction of $P_{e}$ may be reduced to the satisfaction of $P_{e k}^{*}, k \in \mathbb{N}$. Fix $e$. Assume that $A_{e}, B_{e}$ are not $K$-trivial and $P_{e k t}^{*}$ are met for all $k, t$. Then by Proposition 2.11, for each $k$ there are infinitely many $t$ such that the left-hand-side of the implication in $P_{e k t}^{*}$ holds. Since each $P_{e k t}^{*}$ is met, it follows that for each $k$ there are infinitely many $t$ such that $K\left(V_{e}\left\lceil_{n_{k t}}\right)>g\left(n_{k t}\right)+k\right.$. Since the map $(k, i) \mapsto n_{k}(i)$ is computable and increasing in $i$, Lemma 2.3 shows that $V_{e}$ is not $K$-trivial. Hence $\left(\forall k P_{e k}^{*}\right)$ implies $P_{e}$.

The requirement that $D$ is $K$-trivial can be expressed as

$$
\exists c \forall n, K\left(D \uparrow_{n}\right) \leq g(n)+c .
$$


The cost associated with the enumeration of a number $n$ in $D$ at stage $s+1$ of the construction in view of (3.1) is given by

$$
c(n, s)=\sum_{n \leq i \leq s} 2^{-g(i)}
$$

The satisfaction of (3.1) will be achieved by ensuring that the total cost of the enumerations into $D$ is bounded, in other words

$$
\sum_{(n, s) \in I_{D}} c(n, s)<1 \text { where } I_{D}=\{(n, s) \mid n=\min \{x \mid x \in D[s+1]-D[s]\}\} .
$$

The fact that (3.3) implies (3.1) was established in [DHNS03] when $g$ is replaced by the Kolmogorov function $K(n)$ (also see [DH10, Section 11.1] and [Nie09, Section 5.3] for elaborate presentations of this method). The same argument shows that this implication holds when $K(n)$ is replaced by any right-c.e function $f$ such that $\sum_{i} 2^{-f(i)}<1$.

We close this section by providing a condition which implies $R_{e}$ and shows explicitly the required Turing reductions. By the definition of $a_{e}[s]$ it follows that $a_{e}(n)$ (the final position of $\left.a(n)[s]\right)$ is computable from $A_{e}$. Similarly, $b_{e}(n)$ is computable from $B_{e}$. Hence $A_{e} \oplus D$ computes an upper bound of $n \mapsto d_{e}(n)$ (provided that $\mathbb{N}[\langle e, n\rangle] \cap D$ is finite) and the same is true of $B_{e} \oplus D$. The following condition expresses a weak coding of $V_{e}$ into $D$.

$$
R_{e}^{*}: \quad\left(\begin{array}{l}
\text { For all } k, t, s \text { and all } n \in\left[n_{k}(t-1), n_{k}(t)\right) \cap \mathbb{N}^{[k]} \\
n \in V_{e}[s+1]-V_{e}[s] \Rightarrow d_{e}\left(n_{k}(t)\right)[s] \in D[s+1]-D[s]
\end{array}\right)
$$

Condition $R_{e}^{*}$ implies condition $R_{e}$. Indeed, suppose that $R_{e}^{*}$ holds. Then to determine if $n \in V_{e}$ we can first find $k$ such that $n \in \mathbb{N}^{[k]}$ and then find $t$ such that $n \in\left[n_{k}(t-1), n_{k}(t)\right)$. By the definition of $d_{e}\left(n_{k}(t)\right)[s]$ it follows that it changes value only when one of the following holds:

- $a_{e}\left(n_{k}(t)\right)[s]$ changes value and $b_{e}\left(n_{k}(t)\right)[s]$ changes value;

- the current value of $d_{e}\left(n_{k}(t)\right)[s]$ is enumerated into $D$.

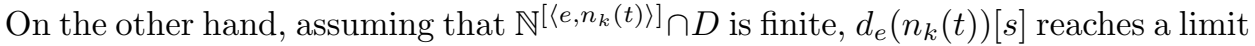
as $s \rightarrow \infty$. Since $a_{e}\left(n_{k}(t)\right)$ is computable from $A_{e}$, it follows that we can use $A_{e} \oplus D$ in order to compute a stage where the approximation to $D[s]\left\lceil_{d_{e}\left(n_{k}(t)\right)[s]+1}\right.$ has reached a limit. By $R_{e}^{*}$, at that stage the approximation to $V_{e}(n)$ has also reached a limit. So we have computed $V_{e}(n)$. The same procedure can be performed via $B_{e} \oplus D$-computations, by first computing $b_{e}\left(n_{k}(t)\right)$. Hence $V_{e}(n)$ is also computable from $B_{e} \oplus D$.

We have established that a construction of $D,\left(V_{e}\right)$ which meets conditions (3.3) and $R_{e}^{*}, P_{e k}$ for $e, k \in \mathbb{N}$ (and any choice of a computable function $(k, i) \mapsto n_{k}(i)$ which is increasing on $i$ and such that $n_{k}(i) \in \mathbb{N}^{[k]}$ ) is sufficient for the proof of Theorem 1.1. An underlying assumption is that for each $e, n$ the set $\mathbb{N}[\langle e, n\rangle] \cap D$ is finite, so that $d_{e}(n)[s]$ reaches a limit. The latter will be an immediate feature of the construction.

3.2. Strategy and witnesses for conditions $P_{e k}^{*}$. Recall that $P_{e k}^{*}$ denotes the conjunction of the conditions $P_{\text {ekt }}^{*}$ of Section 3.1 (which depend on the choice of $\left.(k, i) \mapsto n_{k}(i)\right)$. The construction of Section 3.3 is driven by actions (enumerations into $\left.D, V_{e}\right)$ for the satisfaction of $P_{e k}^{*}$. Here we define some parameters that are used 
in these actions. For each $k$ we define an increasing sequence $\left(n_{k}(i)\right)$ of numbers. Recall the definitions of $(i, j) \mapsto\langle i, j\rangle$ and $\mathbb{N}^{[k]}$ from Section 3.1. Define

$$
J(\langle k, x\rangle)=\left\{\langle k, m\rangle \mid m>x+1 \wedge \sum_{t>\langle k, m\rangle} 2^{-g(t)}>\frac{1}{m-x-1}\right\} .
$$

The sets $J(i)$ are uniformly c.e. and by Lemma 2.4 they are all infinite. Hence we may choose a uniformly computable family of sets $J^{*}(i)$ such that $J^{*}(i) \subseteq J(i)$ for each $i$. Define $\left(n_{k}(i)\right)$ recursively as follows.

$$
\begin{aligned}
n_{k}(-1) & =\min \mathbb{N}^{[k]} \\
n_{k}(i) & =\min J^{*}\left(n_{k}(i-1)\right)
\end{aligned}
$$

Note that the function $(k, i) \mapsto n_{k}(i)$ is computable. Moreover

$$
\sum_{i>n_{k}(t)} 2^{-g(i)}>1 /\left|\left(n_{k}(t-1), n_{k}(t)\right) \cap \mathbb{N}^{[k]}\right| .
$$

From this point on, $P_{e k t}^{*}$ refers to this choice of $(k, i) \mapsto n_{k}(i)$. We say that $P_{e k}^{*}$ requires attention at stage $s+1$ if there is some $t<s$ such that

$$
\sum_{d_{e}\left(n_{k}(t)\right)[s]<i \leq s} 2^{-g(i)}<2^{-e-k} \cdot \sum_{n_{k}(t)<i \leq s} 2^{-g(i)}
$$

and

$$
\forall i \leq p_{e k}[s], K\left(V_{e} \uparrow_{i}\right)[s] \leq g(i)+k
$$

where $p_{e k}[s]$ is the largest stage $\leq s$ where $P_{e k}^{*}$ required attention (and $p_{e k}[s]=0$ if such a stage does not exist). In this case we say that $P_{e k}^{*}$ requires attention for $t$ at stage $s+1$.

The intuition for the main action of the construction is that if (3.5) holds, by enumerating $d_{e}\left(n_{k}(t)\right)[s]$ into $D$ and changing the approximation to $V_{e} \uparrow_{n_{k}(t)}$ the cost of the opponent for maintaining (3.6) is a large multiple of our cost for maintaining (3.3). Our choice of the sequence $\left(n_{k}(i)\right)$ ensures that such attacks are sufficient in order to drive the opponent out of the available descriptions that are needed for maintaining (3.6). Moreover recall that by the analysis of Section 3.1 (which was based on Proposition 2.11) property (3.5) has to hold for infinitely many $t$, if $A_{e}, B_{e}$ are indeed not $K$-trivial.

3.3. Construction of the sets $D, V_{e}$. At stage $s+1$ check if there is some $\langle e, k\rangle<$ $s$ such that $P_{e k}^{*}$ requires attention. If there is such a number, let $\langle e, k\rangle$ be the least one and let $t$ be the least number such that (3.5) and (3.6) hold. Enumerate $d_{e}\left(n_{k}(t)\right)[s]$ into $D$ and enumerate the largest number of

$$
\mathbb{N}^{[k]} \cap\left(n_{k}(t-1), n_{k}(t)\right)-V_{e}[s]
$$

into $V_{e}$.

3.4. Verification of the requirements. At every stage $s+1$ where $P_{e k}^{*}$ requires attention for $t$ and $\langle e, k\rangle<s$, a change in $V_{e} \uparrow_{n_{k}(t)}$ is caused by an enumeration of a number of the set in (3.7) into $V_{e}$ (provided that the set in (3.7) is nonempty). There are

$$
\left|\left(n_{k}(t-1), n_{k}(t)\right) \cap \mathbb{N}^{[k]}\right|
$$


many such enumerations that can be performed. Because of (3.4) and (3.6), each time that $P_{e k}^{*}$ requires attention after such an enumeration, we can count an additional weight of

$$
1 /\left|\left(n_{k}(t-1), n_{k}(t)\right) \cap \mathbb{N}^{[k]}\right|
$$

in the underlying universal prefix-free machine $U$. Since $\operatorname{wgt}(U)<2^{-2}$,

$$
P_{e k}^{*} \text { requires attention less than }\left|\left(n_{k}(t-1), n_{k}(t)\right) \cap \mathbb{N}^{[k]}\right| \text { times for } t .
$$

Hence whenever $P_{e k}^{*}$ requires attention in the construction, an enumeration into $V_{e}$ will occur. Moreover (the current value of) $d_{e}\left(n_{k}(t)\right)[s]$ will only be enumerated into $D$ finitely many times. Marker $d_{e}(i)$ moves at stage $s+1$ only if one of the following events occur:

(a) $A \Upsilon_{i}[s] \nprec A[s+1]$ or $B \Upsilon_{i}[s] \nprec B[s+1]$;

(b) $d_{e}(i)[s] \in D_{e}[s+1]-D_{e}[s]$.

Clearly (a) can only occur at most finitely many times. Moreover (b) only occurs if $i=n_{k}(t)[s]$ for some $t$ such that $P_{e k}^{*}$ requires attention for $t$ at stage $s+1$. By (3.8), case (b) only occurs at most finitely many times. Consequently,

$$
\lim _{s} d_{e}(i)[s] \text { exists for each } e .
$$

In other words $\mathbb{N}^{[\langle e, i\rangle]} \cap D$ is finite, which was an underlying assumption for the requirements of Section 3.1.

Lemma 3.1. For each e, condition $R_{e}$ is met.

Proof. Fix $e$. The construction clearly meets condition $R_{e}^{*}$. By (3.9) and the analysis in Section 3 it follows that $R_{e}$ is met.

Lemma 3.2. For each e, condition $P_{e}$ is met.

Proof. By the discussion of Section 3.1, it suffices to show that $P_{e k t}^{*}$ is met for each $k, t$. Fix $k, t$ and assume that the left hand side of the implication in $P_{e k t}^{*}$ holds. Then according to the construction, (3.8) implies that $K\left(V_{e} \uparrow_{n_{k t}}\right)>g\left(n_{k t}\right)+k$.

Lemma 3.3. The set $D$ is $K$-trivial.

Proof. By the analysis in Section 3.1 it suffices to show (3.3). Let

$$
I_{D}(e, k)=\left\{\left(d_{e}\left(n_{k t}[s-1]\right), s\right) \in I_{D} \mid s, t>0\right\} .
$$

Note that $I_{D}(e, k)$ contains the pairs in $I_{D}$ that correspond to actions for $P_{e k}^{*}$. In particular, $I_{D}=\bigcup_{e, k} I_{D}(e, k)$ and it suffices to show that

$$
\sum_{(n, s) \in I_{D}(e, k)} c(n, s)<2^{-e-k-3}
$$

for each $e, k$. Fix $e, k$ and let $\left(x_{i}, s_{i}\right)$ be a monotone enumeration of $I_{D}(e, k)$, in the sense that $s_{i}<s_{i+1}$ for each $i$. Let us say that at stage $s_{i+1}$ the $i$ th cycle of $P_{e k}^{*}$ is completed. Note that the sequence $\left(x_{i}, s_{i}\right)$ is possibly infinite. However upon the completion of the $i$ th cycle of $P_{e k}$ we may count an additional set of descriptions of the universal machine $U$ (describing current values of $V_{e}$ ) of weight at least $2^{e+k} \cdot c\left(x_{i}, s_{i}\right)$. This is a consequence of (3.5) and (3.6). For the case that $\left(x_{i}, s_{i}\right)$ is finite (so the last cycle is never completed) note that $c\left(x_{i}, s_{i}\right)<2^{-e-k-4}$ for all $i$ due to (3.5). Since $\operatorname{wgt}(U)<2^{-4}$ we obtain $\sum_{i} c\left(x_{i}, s_{i}\right)<2^{-e-k-3}$, i.e. $(3.10)$. 
According to the analysis of Section 3.1, this concludes the proof of Theorem 1.1.

\section{Conclusion}

The class of $K$-trivial sequences and their Turing degrees is far from trivial and, in fact, has very rich structure. There are several ways one can reveal the complexities of this class. One of these is the study of the quotient structure of the c.e. Turing degrees modulo the $K$-trivial degrees. Intuitively, this structure gives information about the degrees of unsolvability of c.e. sets when $K$-trivial information is available 'for free'. The following is a direct consequence of Theorem 1.1.

Corollary 4.1. The quotient upper semi-lattice of the c.e. Turing degrees modulo the $K$-trivial degrees has no minimal pairs.

We do not know much more about this structure; for example, the following basic question is open.

\section{Is the quotient upper semi-lattice of the c.e. Turing degrees} modulo the $K$-trivial degrees dense?

Our result shows that a certain simple definition of the ideal of the $K$-trivial degrees with parameters is not possible in the c.e. degrees. In particular, the $K$-trivial degrees cannot be defined as the intersection of two lower cones in the c.e. Turing degrees. The question of parameter definability of this ideal in the c.e. degrees (briefly discussed in the end of [Nie05]) remains open.

\section{REFERENCES}

[Bar10] George Barmpalias. Elementary differences between the degrees of unsolvability and the degrees of compressibility. Ann. Pure Appl. Logic, 161(7):923-934, 2010.

[Bar11] George Barmpalias. On strings with trivial Kolmogorov complexity. Int J Software Informatics, 5(4):609-623, 2011.

[Bar13] George Barmpalias. Universal computably enumerable sets and initial segment prefixfree complexity. Information and Computation, 2013. (in press).

[BD09] Laurent Bienvenu and Rod Downey. Kolmogorov complexity and Solovay functions. In Susanne Albers and Jean-Yves Marion, editors, STACS, volume 3 of LIPIcs, pages 147-158. Schloss Dagstuhl - Leibniz-Zentrum fuer Informatik, Germany, 2009.

[BL11] George Barmpalias and Andrew E.M. Lewis. Chaitin's halting probability and the compression of strings using oracles. Proceedings of the Royal Society A, 467:29122926, 2011.

[BMN11] Laurent Bienvenu, Wolfgang Merkle, and André Nies. Solovay functions and Ktriviality. In STACS, pages 452-463, 2011.

[BN11] George Barmpalias and André Nies. Upper bounds on ideals in the computably enumerable turing degrees. Ann. Pure Appl. Logic, 162(6):465-473, 2011.

[Cha75] Gregory J. Chaitin. A theory of program size formally identical to information theory. J. Assoc. Comput. Mach., 22:329-340, 1975.

[CHKW01] Christian. Calude, Peter. Hertling, Bakhadyr Khoussainov, and Yongge Wang. Recursively enumerable reals and Chaitin $\Omega$ numbers. Theoret. Comput. Sci., 255(1-2):125149, 2001.

[DH10] Rod Downey and Denis Hirshfeldt. Algorithmic Randomness and Complexity. Springer, 2010.

[DHNS03] Rod G. Downey, Denis R. Hirschfeldt, André Nies, and Frank Stephan. Trivial reals. In Proceedings of the 7th and 8th Asian Logic Conferences, pages 103-131, Singapore, 2003. Singapore Univ. Press.

[HKM09] Rupert Hölzl, Thorsten Kräling, and Wolfgang Merkle. Time-bounded kolmogorov complexity and solovay functions. In Mathematical foundations of computer science, 2009, volume 5734 of Lecture Notes in Comput. Sci., pages 392-402. Springer, 2009. 
[Kol65] Andrey N. Kolmogorov. Three approaches to the definition of the concept "quantity of information". Problemy Peredači Informacii, 1(vyp. 1):3-11, 1965.

[KP54] Stephen C. Kleene and Emil Post. The upper semi-lattice of degrees of recursive unsolvability. Ann. of Math. (2), 59:379-407, 1954.

[KS01] Antonín Kučera and Theodore Slaman. Randomness and recursive enumerability. SIAM J. Comput., 31(1):199-211, 2001.

[KS09] Antonín Kučera and Theodore Slaman. Low upper bounds of ideals. J. Symbolic Logic, 74(2):517-534, 2009.

[Lac66] Alistair H. Lachlan. Lower bounds for pairs of recursively enumerable degrees. Proc. London Math. Soc. (3), 16:537-569, 1966.

[Lev73] Leonid A. Levin. The concept of a random sequence. Dokl. Akad. Nauk SSSR, 212:548-550, 1973.

[LV97] Ming Li and Paul Vitányi. An introduction to Kolmogorov complexity and its applications. Graduate Texts in Computer Science. Springer-Verlag, New York, second edition, 1997.

[Mil10] Joseph S. Miller. The $K$-degrees, low for $K$ degrees, and weakly low for $K$ sets. Notre Dame J. Formal Logic, 50(4):381391, 2010.

[ML66] Per Martin-Löf. The definition of random sequences. Information and Control, 9:602619, 1966.

[MN06] Joseph S. Miller and André Nies. Randomness and computability: open questions. Bull. Symbolic Logic, 12(3):390-410, 2006.

[Nie02] Andre Nies. Reals which compute little. In Logic Colloquium, 2002.

[Nie05] André Nies. Lowness properties and randomness. Adv. Math., 197(1):274-305, 2005.

[Nie09] André Nies. Computability and Randomness. Oxford University Press, 2009.

[Odi89] Piergiorgio G. Odifreddi. Classical recursion theory. Vol. I. North-Holland Publishing Co., Amsterdam, 1989.

[Sho81] Richard A. Shore. The theory of the degrees below 0'. J. London Math. Soc., 24:1-14, 1981.

[Sol64] Ray J. Solomonoff. A formal theory of inductive inference. I and II. Information and Control, 7:1-22 and 224-254, 1964.

[Sol75] Robert Solovay. Handwritten manuscript related to Chaitin's work. IBM Thomas J. Watson Research Center, Yorktown Heights, NY, 215 pages, 1975.

[Spe56] Clifford Spector. On the degrees of recursive unsolvability. Ann. of Math. (2), 64:581$592,1956$.

[Ste11] Tom Sterkenburg. Sequences with trivial initial segment complexity. MSc Dissertation, University of Amsterdam, February 2011.

[Yat66] C.E. Mike Yates. A minimal pair of recursively enumerable degrees. J. Symbolic Logic, 31:159-168, 1966.

George Barmpalias: State Key Lab of Computer Science, Institute of Software, Chinese Academy of Sciences, Beijing 100190, P.O. Box 8718, China.

E-mail address: barmpalias@gmail.com

$U R L:$ http://www. barmpalias.net

Rod G. Downey: School of Mathematics, Statistics and Operations Research, Victoria University, P.O. Box 600, Wellington, New Zealand.

E-mail address: rod.downey@vuw.ac.nz

$U R L:$ http://homepages.ecs.vuw.ac.nz/ ${ }^{\sim}$ downey 